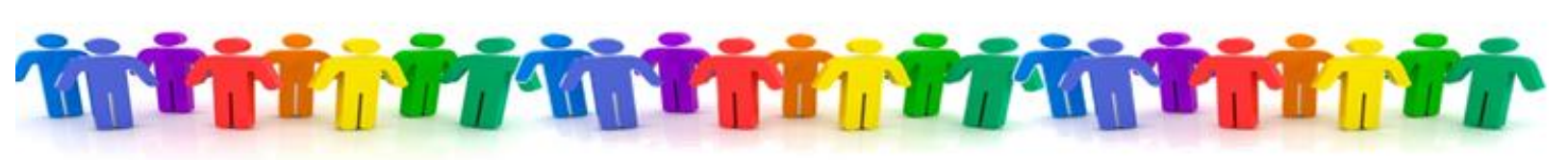

\title{
Binge Drinkers Shouldn't Set Their Own Alcohol Reduction Goals! Evaluating the Effectiveness of Different Goal-Based Alcohol Reduction Interventions Among Young People
}

\author{
Mark Rubin and Alison Hutton \\ The University of Newcastle, Australia
}

Citation: Rubin, M., \& Hutton, A. (2022). Binge drinkers shouldn't set their own alcohol reduction goals! Evaluating the effectiveness of different goal-based alcohol reduction interventions among young people. Alcoholism Treatment Quarterly. https://doi.org/10.1080/07347324.2022.2037486

\begin{abstract}
The present research aimed to investigate the relative effectiveness of three types of alcohol reduction intervention. Participants were 354 university students from an Australian university. After completing an initial survey, they were randomly assigned to either (a) follow national guidelines for alcohol consumption, (b) set their own personal alcohol consumption reduction goal, or (c) work with their peers to set a goal. Participants then recorded their alcohol consumption in a drinking diary over a period of four weeks. The results showed that participants in the personal goal condition reported significantly higher alcohol consumption than those in the national guidelines conditions, with participants in the group goal condition reporting mid-level alcohol consumption. This effect was moderated by binge drinking propensity. Personal goal setting was most likely to lead to higher alcohol consumption when participants scored relatively high on measures of binge drinking propensity. These findings highlight the point that different types of alcohol reduction interventions may be effective for different types of people. In particular, people who have a relatively high propensity for binge drinking should be encouraged to follow goals that have been set by authorities (e.g., national guidelines) rather than by themselves.
\end{abstract}

Keywords: adolescent alcohol reduction; brief interventions; goal setting; adolescent drinking

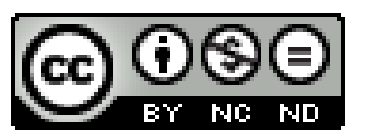

Copyright (C) The Author. OPEN ACCESS: This material is published under the terms of the Creative Commons Attribution-NonCommercial-NoDerivatives 4.0 International licence (CC BY-NC-ND 4.0; https://creativecommons.org/licenses/bync-nd/4.0/). This licence permits you to copy and redistribute this material in any medium or format for noncommerical purposes without remixing, transforming, or building on the material provided that proper attribution to the authors is given.

This self-archived version is provided for non-commercial and scholarly purposes only. We are grateful to Katy Gray and Paula Convery for their assistance with data collection. Correspondence concerning this article should be addressed to Mark Rubin at the Department of Psychology, Durham University, South Road, Durham, DH1 3LE, UK. Tel: +61407949785. E-mail: Mark-Rubin@outlook.com Web: http://bit.ly/rubinpsyc 
Harmful use of alcohol has serious effects on individual physical and mental wellbeing (e.g., Churchill \& Farrell, 2017; Corrao et al., 2004), and it is considered by the World Health Organization (2019) to be one of the main risk factors for poor health globally. However, the risk of harm is experienced disproportionally by young people aged 15-24, whose brains and bodies are still developing and who are more susceptible to alcohol's effects, including cognitive impairment. For example, a recent systematic literature review of 31 longitudinal studies of adolescents and young adults found that alcohol use was associated with abnormal development of gray matter of the brain (de Goede et al., 2021). Drinking less frequently and less on each occasion reduces the lifetime risk of alcohol related harm. Indeed, for people under the age of 18, not drinking alcohol is the safest option (Australian Institute of Health and Welfare, 2021).

Binge drinking is a particular health risk for adolescents and young adults (de Goede et al., 2021), and it is associated with an increased risk of injury from motor vehicle accidents, drowning, and violence, as well as an increase in the incidence of reckless behaviours such as unsafe sex and drunk driving (Hamilton et al., 2018; Jones et al., 2020; Solomon, 2014; Van Gemert et al., 2011; Zador et al., 2000). Binge drinking also increases the likelihood of future alcohol addiction (de Goede et al., 2021; Jennison, 2004) and long-term health consequences such as liver damage, cancer, and dementia (Grønbæk, 2009). Therefore, it is important to test interventions that may help to reduce unsafe drinking behaviour among young people. The aim of this preregistered study was to investigate the relative effectiveness of three types of alcohol reduction interventions among a sample of young Australians, who are more likely than the general Australian population to consume alcohol in excessive quantities (Gilchrist et al., 2012).

Our interventions were based on goal setting, in which people set their own alcohol reduction goals and then attempt to adhere to these goals. We were particularly interested in the source of the alcohol reduction goals, and we contrasted goals that were prescribed by a national authority with goals that were developed by participants themselves either on their own or in consultation with their peer group.

Our preregistered hypotheses were as follows. H1: Participants who set their own personal alcohol consumption reduction goals or who develop their goals together with a group of peers will show a stronger reduction in excessive alcohol consumption compared to participants who are asked to follow the (Australian) National Guidelines to Reduce Health Risks from Drinking Alcohol. In addition, participants who develop their alcohol consumption reduction goals together with a group of peers will show a stronger reduction in excessive alcohol consumption compared to participants who set their own personal alcohol consumption goals.

$\mathrm{H} 2$ : Binge drinking self-efficacy, strength of intention to binge drink, and scores on the Alcohol Use Disorders Identification Test (AUDIT) will moderate the H1 effect of condition on alcohol consumption. The effect will be reduced among people who have high self-efficacy, strong intention to binge drink, and high scores on the AUDIT.

H3: Social group norms and subjective norms for binge drinking will moderate the H1 effect of condition: The effect will be weaker among participants whose social groups are more supportive of binge drinking.

H4: Ingroup identification will moderate the H1 effect of condition: The effect will be stronger among participants who identify more strongly with their ingroup.

H5: There will be an effect of condition on the sustainability of excessive alcohol reduction across the four week assessment period. Personal and group goals will have a more sustained effect across the four week period than National Guideline goals. In addition, group goals will have a more sustained effect than personal goals. 


\section{Method}

The study received ethical approval from the human research ethics committee of the authors' institution. The research method and analyses were preregistered, and the preregistered protocol is available at: https://osf.io/m82nt/?view_only=577561066a3d4fb98aa8631b191d95e6 The research deviated from the preregistered protocol in several ways. We highlight major deviations throughout using the superscript NP (to indicate "not preregistered"). We also acknowledge the limitations that are associated with the practice of preregistration (Rubin, 2020).

\section{Participants and Design}

The data was collected between $13^{\text {th }}$ March 2020 and $28^{\text {th }}$ October 2021 . There were 575 responses to the first survey. Of these, 20 participants had completed either the first or the second survey twice or, in one case, three times (often being assigned to different conditions in the process). These participants were removed from the analyses. Of the remaining 539 participants who attempted the first survey, 520 completed it fully (96.48\%). And, of the 354 participants who then went on to attempt the second survey (34.32\% attrition rate), 341 completed it fully (96.33\%). Missing data was left missing and listwise deletion was used (i.e., participants were excluded from analyses if their data was missing from the variables that were included in an analysis).

In the case of psychology participants, responses from the two surveys were linked via their research participant pool identification number. Non-psychology participants were asked to generate their own unique identifier code. Please note that two non-psychology participants who attempted the second survey did not provide an identifying code that matched with a code in the first survey. These two participants were removed from the analyses.

Of the 354 participants who attempted both surveys, all but one was enrolled in an undergraduate psychology course and participated in exchange for course credit. These 354 participants ranged in age from 17 to 60 years and had a mean age of 22.05 years $(S D=6.58)$. Most identified as "Caucasian/White" $(n=288,81.36 \%)$, with the next highest proportion identifying as "Aboriginal or Torres Strait Islander" $(n=19,5.37 \%)$. With regards to gender, $84.75 \%$ identified as "female" $(n=300)$ and $14.41 \%$ as "male" $(n=51)$. This gender imbalance is typical in psychology courses. Finally, $96.89 \%$ of participants $(n=343)$ indicated that Australia was their country of residence. A comparison between participants who completed the second survey and those who did not found no significant differences in terms of their age, $t(526)=-.023$, $p=.981$; ethnicity, $\chi^{2}(5)=9.74, p=.083$; gender, $\chi^{2}(3)=3.28, p=.351$; and country of residence, $\chi^{2}(1)<.001, p=.987$.

The research used a cross-sectional experimental design, with goal type (national guidelines/personal goal/group goal) as the between-subjects factor. Of the 354 participants who attempted both surveys, 121 (34.18\%) were assigned to the national guidelines condition, 108 $(30.51 \%)$ were assigned to the personal goal condition, and $125(35.31 \%)$ were assigned to the group goal condition. There was no significance difference between the three conditions in the drop-out rate moving from the first to the second survey, $\chi^{2}(2)=.71, p=.703$.

Our final sample size of 354 was smaller than our preregistered goal of 420 . Nonetheless, this sample size had good power (.81) to detect a relatively small effect size $(r=.15)$ using a twotailed correlation test and a conventional significance threshold (.05). 


\section{Procedure and Measures}

The survey introduced the research as investigating the "effectiveness of goal setting to reducing excessive alcohol consumption among young people in Australia." Participants were recruited from a large Australian public university. People were eligible to participate in the research if they were between the ages of 18 and 26 years, could converse in English, were a student at the researchers' university, had access to a computer or smart phone, were not pregnant, were not currently undergoing mental health treatments, and did not self-identify as having an alcohol addiction. Please note that $12.18 \%$ of participants were older than 26 years. We decided to retain these people in our analyses to achieve adequate statistical power. ${ }^{\mathrm{NP}}$ However, the key results remained significant when these people were excluded from the analyses.

Participants completed two online surveys one month apart. They were told that their responses were not anonymous to the researchers but that their identity would remain confidential. The first survey took a median time of 7.22 minutes to complete, and the second survey took a median time of 3.41 minutes. The first survey measured participants' demographic variables (age, gender, ethnic background, country of residence). It also included a series of six measures based on Johnston and White's (2003) study. Participants responded to these measures using a 7-point response scale.

The first two measures assessed identification and sense of belonging with friends and peers. These included a 4-item measure of ingroup identification (e.g., "How much do you feel you identify with your peers?", with response scale anchored not very much to very much) and a 2-item measure of sense of belonging with friends (e.g., "In general, how well do you feel you fit into your groups of friends and peers?", not very well to very well). The next two measures assessed normative considerations. These included a 4-item measure of social group norms (e.g., "How many of your friends and peers would think that drinking five or more standard alcoholic beverages in a single session in the next two weeks is a good thing to do?", none to all) and a 3-item measure of subjective norms for binge drinking (e.g., "Most people who are important to me think that my drinking five or more standard alcoholic beverages in a single session in the next two weeks would be...," undesirable to desirable). Finally, two measures assessed binge drinking propensity. These included a 2-item measure of binge drinking self-efficacy (e.g., "For me to drink five or more standard alcoholic beverages in a single session in the next two weeks would be...," very difficult to very easy) and a 3-item measure of strength of intention to binge drink (e.g., "I intend to drink five or more standard alcoholic beverages in a single session in the next two weeks," extremely likely to extremely unlikely; reverse scored). These six scales all had good internal consistency in the current study (Cronbach alpha or Spearman-Brown coefficients $\geq .76$ ).

At the end of the first survey, participants downloaded a drinking diary. They were asked to record their drinking goals and behaviours for the following month in this diary. At this stage, participants were randomly assigned to an experimental condition. In the national guidelines condition, participants were asked to create a drinking goal following Australia's (2009) National Health and Medical Research Council (NHMRC) Australian Guidelines to Reduce Health Risks from Drinking Alcohol. According to these guidelines, healthy men and women should drink no more than two standard drinks on any day and no more than four standard drinks on any single occasion. The guidelines were developed by a working committee of Australian experts following a review of the relevant literature. The guidelines were peer reviewed by health economists, epidemiologists, and experts in alcohol research and public health. The specific guidelines that were used in this study were intended for healthy male and female adults in Australia. Please note 
that the guidelines were updated in 2020 (NHMRC, 2020). However, for our purposes, the 2020 recommendations remained substantively similar to the 2009 guidelines.

Participants were provided with the following example of a drinking goal: "I will only drink on the weekend, and I will have no more than 4 standard drinks on any single day." Participants were instructed to make their goal specific (e.g., only drink on the weekend), measurable (e.g., 4 standard drinks), achievable (e.g., still permitted to drink on weekend), relevant (e.g., reducing alcohol consumption), and time based (e.g., this month).

Participants in the personal and group goal conditions were also provided with the national guidelines and received similar instructions except they were asked to either "develop your own personal goal" or develop a group goal "working with your friends and people you know, in a group of 2-5 people." Participants in all conditions were asked to write their alcohol reduction goal at the top of their drinking diary and to then record (a) the number of drinking occasions and (b) the number of standard drinks per occasion for each day of the week for four weeks. At the end of the month, participants inputted the information from their drinking diaries to the second survey. Hence, the second survey recorded participants' number of drinking occasions each week during the assessment month and the number of standard drinks per occasion. Given the relatively strong positive correlation between these two measures $(r=.63, p<.001, n=341)$, we decided to multiply participants' number of drinking occasions and number of standard drinks per occasion to create a more reliable index of total alcohol consumption. ${ }^{\mathrm{NP}}$

In a non-preregistered part of our methodology, participants also indicated their drinking goals for each week in the second survey. Critically, participants were not asked to record this information in their drinking diary. Hence, this measure may have suffered from memory recall errors. Participants also completed the 10-item Alcohol Use Disorders Identification Test in both surveys. This instrument helps to identify individuals who are hazardous drinkers or have an existing drinking problem (Bush et al., 1998). The scale had good internal consistency in the two surveys (Cronbach $\alpha \mathrm{s} \geq .72$ ). Participants' mean score on this measure in the first survey was 7.19 $(S D=4.76)$, which placed them in the "low risk" category, but higher than the mean score for a representative sample of the Australian population that was sampled in $2016(M=4.58$; O'Brien et al., 2020).

Finally, participants completed the Perceived Awareness of the Research Hypothesis scale at the end of the second survey (PARH; Rubin, 2016). This 4-item scale measures the extent to which participants believe that they are aware of the research hypotheses (e.g., "I knew what the researchers were investigating in this research"). Participants responded using a 7-point scale anchored strongly disagree and strongly agree. The scale had good internal consistency in the current study (Cronbach $\alpha=.88$ ).

\section{Results}

Please note that, following Rubin (2021) and consistent with our preregistered analysis plan, we undertook single tests of multiple individual null hypotheses (i.e., individual testing) rather than multiple tests of single joint null hypotheses (in which a significant result for any test is sufficient to reject the associated joint null hypothesis; i.e., disjunction testing). As per Rubin's (2021) review, an alpha adjustment is not necessary in this case because we only had one opportunity to make a Type I error in relation to each null hypothesis test. 


\section{The Effect of Condition on Alcohol Consumption}

Our first hypothesis was that participants who set their own personal alcohol consumption reduction goals or who developed their goals together with a group of peers would show a stronger reduction in alcohol consumption compared to participants who were asked to follow the national guidelines, and that participants in the group goal condition would show a stronger reduction compared to those in the personal goal condition. A one-way ANOVA showed a significant effect of condition on total alcohol consumption, $F(2,338)=4.12, p=.017, \eta^{2}=.024$. Please note that there were five outliers on the index of total alcohol consumption (defined as $\pm 3 S D$ s from the sample mean). However, the result of this analysis remained significant when these cases were excluded, $F(2,333)=3.06, p=.048, \eta^{2}=.018$.

As shown in Table 1, least significant difference tests showed that participants in the personal goal condition reported significantly higher alcohol consumption than participants in the national guidelines condition. This result was opposite to our predictions. Participants in the group goal condition also had significantly higher reported alcohol consumption than those in the national guidelines condition, although this effect became nonsignificant when outliers were excluded from the analysis. Hence, the clearest effect was that adherence to the national guidelines lowered reported alcohol consumption relative to adherence to a personal goal.

Table 1

Total Reported Alcohol Consumption as a Function of Condition

\begin{tabular}{lcc}
\hline Condition & Including Outliers & Excluding Outliers \\
\cline { 2 - 3 } National guidelines & $4.58_{\mathrm{a}}(9.88)$ & $4.58_{\mathrm{a}}(9.88)$ \\
Personal goal & $15.79_{\mathrm{b}}(48.92)$ & $8.78_{\mathrm{b}}(17.36)$ \\
Group goal & $7.44_{\mathrm{b}}(18.77)$ & $5.99_{\mathrm{ab}}(9.96)$ \\
\hline
\end{tabular}

Note. Standard deviations are shown in parentheses. Means that do not share any common subscript differ significantly between conditions ( $p s<.05$ ). Note that the large $S D$ in the first column of the personal goal condition is due to the presence of four outliers.

It is possible that the reported results were caused by demand characteristics (Orne, 1962). In particular, it is possible that participants in the national guidelines condition reported the lower alcohol consumption than those in the personal goal condition because they believed that this result would confirm the researchers' hypothesis, and they wanted to please the researchers. To investigate this demand characteristics explanation, we tested the effect of condition on total alcohol consumption including scores from the Perceived Awareness of the Research Hypothesis scale as a covariate. Contrary to the demand characteristics explanation, the effect of condition remained significant in the analysis that included outliers, $F(2,337)=3.46, p=.033$, partial $\eta^{2}=$ .020 . The effect was nonsignificant when outliers were excluded, $F(2,332)=3.00, p=.051$, partial $\eta^{2}=.018$, although the $p$ value was very close to our significance threshold.

\section{Moderators of the Effect of Condition on Alcohol Consumption}

Our second hypothesis was that binge drinking self-efficacy, strength of intention to binge drink, and AUDIT score (from the first survey) would moderate the effect of condition on alcohol consumption, with personal and group goals being the least effective at reducing alcohol consumption among people who had high self-efficacy, strong intention to binge drink, and high AUDIT scores. We tested these potential moderation effects using Hayes' (2018) PROCESS software. The continuous variables were mean centred prior to computing interaction terms. Indicator effects coding was used to examine (a) the effect of personal goal condition relative to 
the other two conditions combined and (b) the effect of group goal condition relative to the other two conditions combined. These conditional effects were examined at the $16^{\text {th }}, 50^{\text {th }}$, and $84^{\text {th }}$ percentiles of the putative moderator variable. For brevity, we only report the results of analyses that excluded the five outliers on the total alcohol consumption index. However, the substantive conclusions did not change when these outliers were included in the analyses.

There was a significant overall interaction between binge drinking self-efficacy and condition, $F(2,329)=4.64, p=.010$ (i.e., the highest order unconditional interaction effect). Binge drinking self-efficacy did not significantly moderate the effect of the group goal condition, $b=$ 0.37 , $s e=0.75, t=0.49, p=.728$. However, it did significantly moderate the effect of the personal goal condition, $b=2.47$, se $=0.85, t=2.89, p=.004$. The effect of personal goal condition was nonsignificant at low levels of binge drinking self-efficacy, $b=-2.51$, se $=2.86, t=-0.88, p=.380$, significant and larger at medium levels, $b=4.89$, $s e=1.64, t=2.97, p=.003$, and significant and largest at high levels, $b=9.82, s e=2.52, t=3.89, p<.001$. Note that the coefficient was positive in these latter two cases, indicating that, contrary to predictions, participants with medium or high binge drinking self-efficacy reported consuming more alcohol in the personal goal condition than in the other two conditions combined.

A similar pattern of results was found for intention to binge drink. The overall interaction with condition was significant, $F(2,329)=12.94, p<.001$. Intention to binge drink did not significantly moderate the effect of the group goal condition, $b<0.01$, se $=0.66, t=0.01, p=.994$. However, it did significantly moderate the effect of the personal goal condition, $b=3.15$, se $=$ $0.71, t=4.43, p<.001$. This effect was nonsignificant at low levels of intention to binge drink, $b$ $=-1.89$, $s e=2.14, t=-0.88, p=.378$, and at medium levels, $b=2.31$, $s e=1.63, t=1.42, p=.157$, but significant at high levels, $b=13.86$, $s e=2.57, t=5.40, p<.001$. Hence, participants with high intention to binge drink reported consuming more alcohol in the personal goal condition than in the other two conditions combined.

Finally, a similar pattern of results emerged for the AUDIT. The overall interaction with condition was significant, $F(2,330)=8.511, p<.001$. AUDIT scores did not significantly moderate the effect of the group goal condition, $b=-0.17$, se $=0.32, t=-0.52, p=.606$, but they did significantly moderate the effect of the personal goal condition, $b=1.02$, se $=0.32, t=3.18, p$ $=.002$. The effect of personal goal condition was nonsignificant at low AUDIT scores, $b=-1.62$, $s e=2.00, t=-0.81, p=.420$, and at medium levels, $b=1.44$, se $=1.55, t=0.93, p=.353$, but significant at high levels, $b=7.55$, $s e=2.18, t=3.46, p<.001$. Hence, participants with high AUDIT scores reported consuming more alcohol in the personal goal condition than in the other two conditions combined.

We also predicted that social group norms and subjective norms for binge drinking would moderate the effect of condition such that it would be weaker among participants whose social groups are more supportive of binge drinking. There was no significant overall interaction between social group norms and condition, either with or without outliers $(p s \geq .342)$. There was a significant overall interaction between subjective norms for binge drinking and condition $(p=$ .040). However, this effect became nonsignificant when outliers were excluded $(p=.301)$, and so we did not investigate it further. Hence, normative concerns did not appear to have a significant influence on the effect of condition.

We also investigated the potential moderating effect of ingroup identification and sense of belonging with friends. Again, these results were inconsistent. There was no significant overall interaction between social group norms and condition, either with or without outliers ( $p s \geq .179)$. 
There was a significant overall interaction between ingroup identification and condition when outliers were included $(p=.003)$ but not when they were excluded $(p=.074)$.

Our final hypothesis was that personal and group goals would have a more sustained effect across the four week period than National Guideline goals, and that group goals would have the most sustained effect. To test this prediction, we conducted a 3 (condition: personal goal/group goal/national guidelines) x 4 (week: 1/2/3/4) mixed-model ANOVA with repeated measures on the second factor on total alcohol consumption. The condition by weeks interaction effect was nonsignificant (with outliers: $p=.757$; without outliers: $p=.779$ ), indicating that the effect of condition did not vary significantly over the four week period.

\section{The Effect of Condition on Drinking Goals}

Recall that, in a non-preregistered part of our methodology, we asked participants to indicate their drinking goals for each week. We had not preregistered any hypotheses about this measure, and we had some doubts about its reliability given that participants were not asked to record this information in their drinking diaries, and so they may have had difficulty recalling this information. Consistent with these concerns, there was a relatively large amount of missing data on this measure ( $n=157 ; 44.35 \%$ ). Nonetheless, we proceeded to test the effect of condition on total drinking goals (i.e., goals for number of drinking occasions multiplied by goals for number of standard drinks per occasion). ${ }^{\mathrm{NP}}$ The one-way ANOVA showed no significant effects either including outliers, $F(2,194)=0.34, p=.714, \eta^{2}=.003$, or excluding outliers, $F(2,190)=0.06, p$ $=.946, \eta^{2}=.001$.

\section{Discussion}

The present study examined the effect of three goal-based alcohol reduction intervention strategies among a group of Australian university students. Contrary to our predictions, participants who set their own alcohol reduction goals subsequently reported significantly higher alcohol consumption than those who followed national guidelines. Put another way, adherence to the national guidelines lowered reported alcohol consumption relative to adherence to a personal goal. This effect did not appear to be driven by demand characteristics.

The effect of condition was moderated by binge drinking propensity. We had predicted that personal and group goals would be least effective at reducing alcohol consumption among people who had high self-efficacy, strong intention to binge drink, and a high score on the AUDIT. This prediction was not confirmed for the group goal condition. However, it was partially confirmed for the personal goal condition. In particular, participants with medium or high binge drinking selfefficacy, high intention to binge drink, and high AUDIT scores all reported consuming a greater amount of alcohol in the personal goal condition than in the other two conditions. Hence, consistent with our prediction, the personal goal condition was least effective at reducing alcohol consumption for these participants. However, what we had not expected to find was that this condition was only effective among these participants, and that it was effective in increasing their alcohol consumption relative to the two other conditions. Although unexpected, this evidence is important because it highlights how certain types of alcohol reduction interventions may potentially backfire for certain types of people. In particular, it appears that when people who are at a higher risk of engaging in binge drinking are asked to set their own personal goal to reduce their alcohol consumption, they consume alcohol at a higher level to those who are asked to comply with national guidelines or a group-based goal. Hence, a key implication of the present research findings is that people who are at a higher risk of engaging in binge drinking should not be 
encouraged to set their own personal alcohol reduction goals. In other words, as per our title, binge drinkers shouldn't set their own alcohol reduction goals! Instead, they should be encouraged to follow goals that have been set by authorities (e.g., national guidelines) rather than by themselves.

Interestingly, normative considerations, ingroup identification, and sense of belonging did not reliably moderate the effect of condition on alcohol consumption. In particular, these variables did not moderate the increased alcohol consumption that was reported in the personal goal condition. It is difficult to draw firm conclusions from null results. Nonetheless, these findings suggest a type of matching effect insomuch as personal propensities for binge drinking affected personal goals and social and normative considerations did not.

In conclusion, the current findings highlight the importance of taking individual differences into account when designing alcohol reduction interventions. Different types of alcohol reduction interventions may be more or less effective for different types of people. In the present study, we found that people with a relatively high propensity for binge drinking had the highest levels of alcohol consumption when they were asked to set their own alcohol reduction goals. It appears that a more effective intervention approach for these types of people would be to encourage them to follow goals that have been set by authorities (e.g., national guidelines) rather than by themselves.

\section{References}

Australian Institute of Health and Welfare. (2021). Alcohol, tobacco and other drugs in Australia. Australian Government. https://www.aihw.gov.au/reports/alcohol/alcohol-tobacco-otherdrugs-australia/contents/priority-populations/younger-people

Bush, K., Kivlahan, D., Fihn, S., \& Bradley, K. (1998). The AUDIT alcohol consumption questions (AUDIT-C): An effective brief screening test for problem drinking. Alcohol Use Disorders Identification Test, Archives of Internal Medicine, 158(16), 1789-1795. https://doi.org/10.1001/archinte.158.16.1789

Churchill, S. A., \& Farrell, L. (2017). Alcohol and depression: Evidence from the 2014 health survey for England. Drug and Alcohol Dependence, 180, 86-92. https://doi.org/10.1016/j.drugalcdep.2017.08.006

Corrao, G., Bagnardi, V., Zambon, A., \& La Vecchia, C. (2004). A meta-analysis of alcohol consumption and the risk of 15 diseases. Preventive Medicine, 38(5), 613-619. https://doi.org/10.1016/j.ypmed.2003.11.027

de Goede, J., van der Mark-Reeuwijk, K. G., Braun, K. P., le Cessie, S., Durston, S., Engels, R. C., ... \& Oosterlaan, J. (2021). Alcohol and brain development in adolescents and young adults: a systematic review of the literature and advisory report of the health council of the Netherlands. Advances in Nutrition, 12(4), 1379-1410. https://doi.org/10.1093/advances/nmaa170

Gilchrist, H., Smith, K. K., Magee, C., \& Jones, S. (2012). A hangover and a one-night stand: Alcohol and risky sexual behaviour among female students at an Australian university. Youth Studies Australia, 31(2), 35 - 43.

Grønbæk, M. (2009). The positive and negative health effects of alcohol and the public health implications. Journal of Internal Medicine, 265, 407-420. https://doi.org/10.1111/j.13652796.2009.02082.x

Hamilton, K., Keech, J. J., Peden, A. E., Hagger, M. S. (2018). Alcohol use, aquatic injury, and unintentional drowning: A systematic literature review. Drug and Alcohol Review, 37, 752-773. https://doi.org/10.1111/dar.12817 
Hayes, A. F. (2018). Introduction to mediation, moderation, and conditional process analysis: A regression-based approach (2nd ed.). Guildford Press.

Jennison, K. M. (2004). The short-term effects and unintended long-term consequences of binge drinking in college: a 10-year follow-up study. The American Journal of Drug and Alcohol Abuse, 30(3), 659-684.

Johnston, K. L., \& White, K. M. (2003). Binge-drinking: A test of the role of group norms in the theory of planned behaviour. Psychology and Health, 18(1), 63-77. https://doi.org/10.1080/0887044021000037835

Jones, R. M., Van Den Bree, M., Zammit, S., \& Taylor, P. J. (2020). Change in the relationship between drinking alcohol and risk of violence among adolescents and young adults: A nationally representative longitudinal study. Alcohol and Alcoholism, 55(4), 439-447. https://doi.org/10.1093/alcalc/agaa020

National Health and Medical Research Council. (2009). Australian guidelines to reduce health risks from drinking alcohol. https://www.nhmrc.gov.au/sites/default/files/documents/reports/alcohol-harm-reductionfaq.pdf

National Health and Medical Research Council. (2020). Australian guidelines to reduce health risks from drinking alcohol. https://www.nhmrc.gov.au/about-us/publications/australianguidelines-reduce-health-risks-drinking-alcohol

O'Brien, H., Callinan, S., Livingston, M., Doyle, J. S., \& Dietze, P. M. (2020). Population patterns in Alcohol Use Disorders Identification Test (AUDIT) scores in the Australian population; 2007-2016. Australian and New Zealand Journal of Public Health, 44(6), 462-467. https://doi.org/10.1111/1753-6405.13043

Orne, M. (1962). On the social psychology of the psychology experiment: With particular reference to demand characteristics and their implications. American Psychologist, 17, 776 783. https://doi.org/10.1037/h0043424

Rubin, M. (2016). The Perceived Awareness of the Research Hypothesis Scale: Assessing the influence of demand characteristics. Figshare. http://dx.doi.org/10.6084/m9.figshare.4315778

Rubin, M. (2020). Does preregistration improve the credibility of research findings? The Quantitative Methods for Psychology, 16(4), 376-390. https://doi.org/10.20982/tqmp.16.4.p376

Rubin, M. (2021). When to adjust alpha during multiple testing: A consideration of disjunction, conjunction, and individual testing. Synthese, 199, 10969-11000. https://doi.org/10.1007/s11229-021-03276-4

Solomon, S. (2014). The association between heavy episodic drinking and alcohol-related unsafe sex among canadian undergraduate student drinkers. https://ir.lib.uwo.ca/etd/2370

Van Gemert, C., Dietze, P., Gold, J., Sacks-Davis, R., Stoove, M., Vally, H., \& Hellard, M. (2011). The Australian national bing drinking campaign: campaign recognition among young people at a music festival who report risky drinking. BMC Public Health, 11, 482-490. https://doi.org/10.1186/1471-2458-11-482

World Health Organization. (2019). Global status report on alcohol and health 2018. World Health Organization. https://www.who.int/publications/i/item/9789241565639

Zador, P. L., Krawchuk, S. A., Voas, R. B. (2000). Alcohol-related relative risk of driver fatalities and driver involvement in fatal crashes in relation to driver age and gender: An update 
using 1996 data. Journal of Studies on Alcohol and Drugs, 61, 387-395. https://doi.org/10.15288/jsa.2000.61.387

\section{Funding}

Conflict of Interest

We are grateful to the Hunter Medical Research We declare no conflict of interest.

Institute for their financial support. 